\title{
ADAM10 promotes the proliferation of ligamentum flavum cells by activating the PI3K/AKT pathway
}

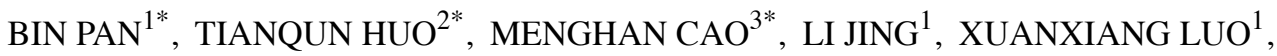

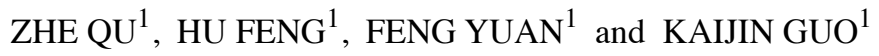 \\ ${ }^{1}$ Department of Orthopedics, The Affiliated Hospital of Xuzhou Medical University, Xuzhou, Jiangsu 221000; \\ ${ }^{2}$ Department of Orthopedics, The First People's Hospital of Lianyungang, Lianyungang, Jiangsu 222061; \\ ${ }^{3}$ Department of Oncology, The Affiliated Hospital of Xuzhou Medical University, Xuzhou, Jiangsu 221000, P.R. China
}

Received June 26, 2020; Accepted October 30, 2020

DOI: $10.3892 /$ ijmm.2020.4809

\begin{abstract}
Ligamentum flavum hypertrophy (LFH) is an important cause of spinal canal stenosis and posterior longitudinal ligament ossification. Although a number of studies have focused on the mechanisms responsible for $\mathrm{LFH}$, the cellular mechanisms remain poorly understood. The aim of the present study was to investigate the roles of differentially expressed genes (DEGs) in LFH, elucidate the mechanisms responsible for LFH and provide a potential therapeutic target for further studies. The GSE113212 dataset was downloaded from the Gene Expression Omnibus (GEO) database. The microarray data were analyzed and DEGs were obtained. Bioinformatics methods, such as Gene Ontology (GO), Kyoto Encyclopedia of Genes and Genomes (KEGG) enrichment and protein-protein interaction (PPI) network analyses were used to obtain the key genes and signaling pathways. In addition, cells derived from hypertrophied ligamentum flavum were cultured, and the key genes and signaling pathways in ligamentum cells were identified through in vitro cell biology and molecular biology experiments. A total of 2,123 genes were screened as DEGs. Among these DEGs, 1,384 genes were upregulated and 739 genes were downregulated. The KEGG pathway analysis revealed that the DEGs were mainly enriched in the PI3K/AKT signaling pathway, and the PPI network analysis screened A disintegrin and metalloproteinase 10 (ADAM10) as a key gene. In vitro experimental verification revealed that ADAM10 promoted the proliferation of ligamentum flavum cells and led to the hypertrophy of the ligamentum by
\end{abstract}

Correspondence to: Professor Kaijin Guo or Professor Feng Yuan, Department of Orthopedics, The Affiliated Hospital of Xuzhou Medical University, 99 Huaihai Road, Quanshan, Xuzhou, Jiangsu 221000, P.R. China

E-mail: guokaijinxz@yeah.net

E-mail: yuanfengxz@yeah.net

*Contributed equally

Key words: ligamentum flavum hypertrophy, A disintegrin and metalloproteinase 10, PI3K/AKT signaling pathway activating the PI3K/AKT pathway. On the whole, the in vitro experimental results suggested that ADAM10 promoted the proliferation of ligamentum flavum cells by activating the PI3K/AKT pathway, which may represent a pathogenic mechanism of LFH. The findings of the present study may provide a basis and direction for further studies on the cellular mechanisms of LFH and present a potential novel therapeutic target and clinical approach.

\section{Introduction}

Ligamentum flavum (LF) hypertrophy (LFH) is an important cause of spinal canal stenosis and posterior longitudinal ligament ossification, which are diseases that lead to sensory and motor impairments in the upper and lower limbs due to the compression of the spinal cord and spinal nerve root or cauda equina nerve $(1,2)$. Spinal stenosis and posterior longitudinal ligament ossification are common spinal diseases affecting the elderly, and Ciol et al estimated that by the year 2025, the number of such cases will reach 64 million (3).

In normal ligamentum tissue, $60-70 \%$ of the extracellular matrix (ECM) consists of elastic fibers, while only $20 \%$ is composed of collagen fibers. In the LF, elastic fibers are arranged neatly and parallel to the long axis and the ECM is filled with a higher amount of collagen fibers and ligamentum cells. When the ligamentum has a high proportion of elastic fibers, the ligamentum presents high elasticity and flexibility, which can maintain the stability of the spine. Conversely, the hypertrophied LF tissue exhibits a loss of elastic fibers, an increased presence of collagen fibers, calcification, ossification and dysplasia of the cartilage (4-6). Losing its unique strength and elasticity, the LF becomes wrinkled and further protrudes into the spinal canal, which leads to various symptoms, such as lumbar spinal stenosis (7).

Researchers have suggested that the repeated mechanical stretching of the LF caused by degenerative lumbar spine instability will cause the elastic fibers to become broken and disordered, and can ultimately lead to a sharp decrease in the number of elastic fibers (6). It has been reported that this repeated mechanical stretching may cause ligamentum damage and lead to fibrotic scars and hypertrophy of the ligaments, which are the main reasons for LFH. However, 
studies have also reported that mechanical stress is only the initiating factor that causes LFH. Nakatani et al (8) conducted continuous mechanical stretching of LF cells and found that LF cells secreted a large amount of transforming growth factor- $\beta 1$ (TGF- $\beta 1$ ) during the stretching process, with the secreted amount presenting a linear relationship with the stress intensity. Further experiments found that TGF- $\beta 1$ induced the synthesis of type I and type III collagen fibers, which leads to LH. Therefore, LF cells may play an important role in LFH.

A number of studies have also reported that the proliferation of LF cells may lead to the secretion of numerous inflammatory factors, such as cyclooxygenase (COX)-2, tumor necrosis factor (TNF)- $\alpha$, interleukin (IL)-1 $\beta$, IL- 8 , IL- $1 \alpha$ and IL- 6 which causes the formation of scar tissue $(4,5,9,10)$. Sairyo et al reported that the formation of scar tissue caused by inflammatory reactions was one of the important causes of LFH and that the degree of hypertrophy was closely related to the degree of fibrosis $(11,12)$. In addition, studies have demonstrated that LF cells can promote fibrin proliferation, which induces the accumulation of collagen fibers and ultimately leads to $\mathrm{LFH}(5,13,14)$.

However, the precise cellular mechanisms of LF cell proliferation and LFH remain unclear $(6,9)$. Thus, the present study analyzed the mRNA expression profiles of the hypertrophic and normal LF, and explored the possible mechanisms responsible for LFH through bioinformatics analyses. In addition, the mechanism responsible for LFH were further validated by molecular biology experiments. On the whole, the results of the present study enhance the current understanding of $\mathrm{LFH}$, provide a direction for further studies clarifying the mechanisms of LFH, and provide potential therapeutic targets for diseases caused by LFH, such as spinal stenosis and ossification of the posterior longitudinal ligament.

\section{Materials and methods}

Collection of microarray data. mRNA microarray data were downloaded from the Gene Expression Omnibus (GEO) database (http://www.ncbi.nlm.nih.gov/geo/) under the accession number GSE113212. An Agilent-039494 SurePrint G3 Human GE v2 8x60K Microarray 039381 (Probe Name version; Agilent Technologies, Inc.) platform was used. A total of 4 LFH samples derived from elderly individuals and 4 non-hypertrophic specimens from young individuals were included in this dataset.

Data preprocessing and differentially expressed gene (DEG) screening. The limma $\mathrm{R}$ package (version 3.36.5) of Bioconductor 3.8 (https://www.bioconductor.org/packages/release/bioc/html/limma.html) was adopted to conduct the quantile normalization of the raw data and subsequent data processing to identify the DEGs between the LFH samples and the normal controls, as previously described $(5,14)$. The DEGs between the 2 groups were evaluated using t-tests, and the P-values were corrected for the false discovery rate (FDR) by the Benjamini-Hochberg $(\mathrm{BH})$ procedure. Only genes with a $\log _{2}$ fold change $(F C) \mid>1$ and FDR $<0.05$ were selected. Volcano plot filtering was applied to visualize the significant DEGs. The differential gene expression patterns between the two sample groups was analyzed by hierarchical clustering.
Functional and pathway enrichment analysis of the DEGs. clusterProfiler V3.8 is a Bioconductor-dependent R software package that not only automates the biological term classification process and gene cluster enrichment analysis, but also provides a visualization module that displays the results of the analysis (15). In the present study, the clusterProfiler package was used for Gene Ontology (GO) and Kyoto Encyclopedia of Genes and Genomes (KEGG) enrichment analyses of the identified DEGs.

Protein-protein interaction (PPI) network. The STRING database (www.string-db.org) is an online database that has been designed to identify PPI pairs and construct PPI networks from large functional groups of proteins. The PPIs obtained for the DEGs were searched using the STRING database, and PPI pairs with a combined score $>0.9$ were selected. A PPI network of the DEGs was then constructed using Cytoscape software (16-18). In the network, each node is a protein and the number of edges corresponds to the degree of interaction. CytoNCA V2.16 is a Cytoscape plugin for network centrality analysis that can be used to identify critical nodes (genes) in a network (19). In the present study, critical genes were identified based on 4 typical centrality measures as follows: Eigenvector centrality, degree centrality, betweenness centrality and closeness centrality. Finally, the top 10 genes in the PPI network were identified as critical genes according to their centrality values.

Isolation, culture and identification of ligamentum cells. According to a previously described method, ligamentum cells were isolated and cultured from patients with lumbar spine stenosis (20). The study was approved by the Ethics Committee of Xuzhou Medical University Affiliated Hospital and written consent was obtained from all patients. Briefly, fresh LF tissue was washed with PBS, cut and digested with type I collagenase, and the digested tissue sections were then plated in a culture dish, as previously described (21). The dish was placed into an incubator for cultivation $\left(5 \% \mathrm{CO}_{2}\right.$, $37^{\circ} \mathrm{C}$ ). After $8 \mathrm{~h}$, DMEM containing fetal bovine serum was added to the culture dish and culturing was continued. When the primary cells migrated out and grew to $80 \%$ confluence, they were digested and passaged with cell digestion solution (trypsin + EDTA). LF cells were cultured in a cell culture incubator containing $5 \% \mathrm{CO}_{2}$ at a constant temperature of $37^{\circ} \mathrm{C}$ in RPMI-1640 medium containing $10 \%$ fetal bovine serum, $50 \mu \mathrm{g} / \mathrm{ml}$ penicillin and $50 \mu \mathrm{g} / \mathrm{ml}$ streptomycin. The cultured cells were observed for fibroblast morphology and identified by vimentin immunostaining and DAPI staining. For immunofluorescence analysis, monolayers of cells were grown on glass coverslips inserted in 6-well plates. After rinsing with PBS, the cells were fixed in $4 \%$ paraformaldehyde in PBS for $10 \mathrm{~min}$ and permeabilized with PBS containing $0.5 \%$ Triton X-100 for $5 \mathrm{~min}$ at room temperature. Blocking was performed with $5 \%$ bovine serum albumin (BSA) in PBS for $30 \mathrm{~min}$, and the cells were subsequently incubated overnight at $4^{\circ} \mathrm{C}$ with anti-vimentin monoclonal antibody (dilution 1:100; \#5741, Cell Signaling Technology, Inc.). Following 3 washes in PBS (5 min each), the cells were incubated for $60 \mathrm{~min}$ at room temperature with a 1:100 dilution of the FITC-conjugated goat anti-rabbit IgG (dilution 1:500; \#4412, Cell Signaling Technology, Inc.) 
respectively. After washing with sterile PBS, the cell nuclei were stained with DAPI, followed by mounting on coverslips using mounting solution overnight at room temperature. Images were obtained and analyzed using the LSM-510 laser confocal microscope system (Carl Zeiss CMP GmbH).

RNA transfection. The sequences of the target gene A disintegrin and metalloproteinase 10 (ADAM10) and a corresponding empty plasmid were obtained from Sangon Biotech (Shanghai) Co., Ltd. The sequence of the human ADAM10 siRNA was 5'-AGACAUUAUGAAGGAUUAUTT-3', and that of the negative control siRNA was 5'-AGGUAGUGUAAU CGCCUUGTT-3'. LF cells were seeded in 24-well plates and transfected when the cell confluence reached $30-50 \%$. Cell transfection was then performed using $5 \mu 1$ Lipofectamine 2000 (Invitrogen; Thermo Fisher Scientific, Inc.) according to the manufacturer's instructions, and $5 \mu \mathrm{l}$ each of ADAM10 siRNA and negative control siRNA to were prepared to a final concentration of $100 \mathrm{nM}$; the transfected cells were collected $48 \mathrm{~h}$ later and used to detect the protein of interest and applied in subsequent experiments. The groups of untreated LF cells, negative control siRNA-transfected cells and ADAM10 siRNA-transfected cells were named the controll, sham1 and ADAM10(-) groups, respectively.

ADAM10 overexpression. LF cells were collected and treated with recombinant lentivirus containing pcDNA3.1-ADAM10 or negative control lentivirus [Sangon Biotech (Shanghai) Co., Ltd.], and the medium was changed following $24 \mathrm{~h}$ of culture. The infection efficiency was $>90 \%$, which was acceptable and indicated that the cells could be used in subsequent experiments. The groups of cells treated with recombinant lentiviruses containing pcDNA3.1-ADAM10 and negative control lentiviruses were named the ADAM10(+) group and the sham 2 group, respectively, and a group of cells without lentivirus treatment was named the control2 group.

Reverse transcription-quantitative PCR (RT-qPCR). All groups of cells were subjected to RT-qPCR.TRIzol (Invitrogen; Thermo Fisher Scientific, Inc.) was added to each culture dish containing cells ( $1 \mathrm{ml}$ of TRIzol per $10^{6}$ cells). According to Universal column RNA extraction kit (CWY065, CWBio), total RNA was extracted and subjected to reverse transcription in a total volume of $20 \mu 1$ to obtain cDNA. qPCR (using the UltraSYBR One Step RT-qPCR kit, CW0659, CWBio) amplification was performed using $0.5 \mu 1$ of cDNA as a template. The reaction conditions are as follows: $95^{\circ} \mathrm{C}$ for $2 \mathrm{~min}, 85^{\circ} \mathrm{C}$ for $15 \mathrm{sec}$ and $60^{\circ} \mathrm{C}$ for $2 \mathrm{~min} \mathrm{sec}, 40$ cycles. A total of $1.5 \mu \mathrm{l}$ of the ADAM10 upstream and downstream primers (5'-CTG CCCAGCATCTGACCCTAA-3' and 5'-TTGCCATCAGAA CTGGCACAC-3'), mix $10 \mu 1$, and water to a total volume of $20 \mu 1$ were used for PCR. $\beta$-actin (5'-GATCATTGCTCC TCCTGAGC-3' and 5'-ACTCCTGCTTGCTGATCCAC-3') served as the internal reference for each group. The data were calculated using the $2^{-\Delta \Delta \mathrm{Cq}}$ method (22).

Western blot analysis. Total protein was extracted by the lysis of all groups of cells with RIPA lysis buffer (Sangon Biotech, Shanghai, China) (including a protease inhibitor and phosphatase inhibitor), and using a BCA kit (Thermo Fisher
Scientific, Inc.) to determine the protein concentration. A total of $10 \mu \mathrm{g}$ protein/lane was separated by $10 \%$ SDS-PAGE and transferred to PVDF membranes. The membranes were blocked with $5 \%$ blocking buffer (BSA) for $1 \mathrm{~h}$ at room temperature and incubated with primary antibodies against ADAM10 (dilution 1:1,000; \#14194, Cell Signaling Technology, Inc.), AKT (dilution 1:1,000; \#9272, Cell Signaling Technology, Inc.), PI3K (dilution 1:1,000; \#4255, Cell Signaling Technology, Inc.), p-AKT (dilution 1:1,000; \#4060, Cell Signaling Technology, Inc.) and p-PI3K (dilution 1:1,000; \#4228, Cell Signaling Technology, Inc.) at $4^{\circ} \mathrm{C}$ overnight. The membranes were then incubated with horseradish peroxidase-conjugated secondary antibodies (dilution 1:1,000; goat anti-mouse IgG, ab205719 and goat anti-rabbit $\mathrm{IgG}, \mathrm{ab205718}$; Abcam) for $1 \mathrm{~h}$ at room temperature and finally developed with an enhanced chemiluminescence (ECL) kit for visualization. The western blot analysis data were analyzed performed using ImageJ software (version 6.0; Media Cybernetics, Inc.).

Cell proliferation analysis. To further detect the role of the ADAM10 gene in the progression of LFH, a PI3K/AKT pathway agonist (740 Y-P, $10 \mu \mathrm{g} / \mathrm{ml}$ ) (HY-P0175, MedChemExpress) was added to the ADAM10(-) cells to form the ADAM10(-)-740 Y-P group, and incubated for 0, 24, 48, 72 and $96 \mathrm{~h}$ at $37^{\circ} \mathrm{C}$. A PI3K/AKT pathway inhibitor (LY294002, $10 \mu \mathrm{M}$ ) (HY-10108, MedChemExpress) was added to the ADAM10(+) cells to form the ADAM10(+)-LY294002 group, and incubated for $0,24,48,72$ and $96 \mathrm{~h}$ at $37^{\circ} \mathrm{C}$. The cells were divided into 5 groups as follows: The control group, ADAM10(+) group, ADAM10(-) group, ADAM10(-) + 740Y-P group and ADAM10(+)-LY294002 group. An EdU uptake assay was performed according to the EdU Imaging kit instructions (Invitrogen; Thermo Fisher Scientific, Inc.). Briefly, equal numbers of cells were incubated with $50 \mathrm{mM}$ EdU for $2 \mathrm{~h}$ at $37^{\circ} \mathrm{C}$. The cells were then subjected to EdU staining by fixation with $4 \%$ paraformaldehyde for $15 \mathrm{~min}$ and permeabilization with 5\% Triton X-100 at room temperature for $20 \mathrm{~min}$. Images of 5 randomly selected non-overlapping regions from each group were collected under a fluorescence microscope (Bio-Rad Laboraroties, Inc.) at a magnification of $\mathrm{x} 200$, and the proportions of EdU-positive cells among DAPI-positive cells were calculated. For the Cell Counting Kit-8 (CCK8) experiment, cells were plated in 6-well plates (cells/well) in a single cell suspension at a concentration of $3 \times 10^{4}$ cells $/ \mathrm{ml}$. Following cell adherence, $10 \mu \mathrm{l}$ of CCK8 reagent were added to each well and incubated for $0,24,48,72$ and $96 \mathrm{~h}$ at $37^{\circ} \mathrm{C}$, after which the OD at $450 \mathrm{~nm}$ was detected using microplate reader (Bio-Rad Laboraroties, Inc.).

Statistical analysis. All experimental data were statistically analyzed by SPSS 19.0 software. The data are expressed as the means \pm standard deviation. In total, 3 independent experiments were performed for RT-qPCR analysis, western blot analysis and CCK- 8 cell proliferation assay. Comparisons between 2 groups were analyzed using the Student's t-test. Multiple sets of groups were analyzed with one-way analysis of variance (ANOVA) with the Fishers' Least Significant Difference post hoc test. P-values of $<0.05$ were considered to indicate statistical significant differences. 
A

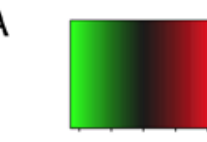

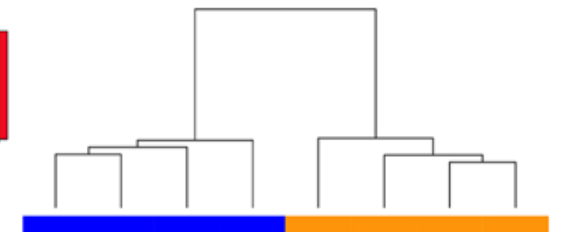

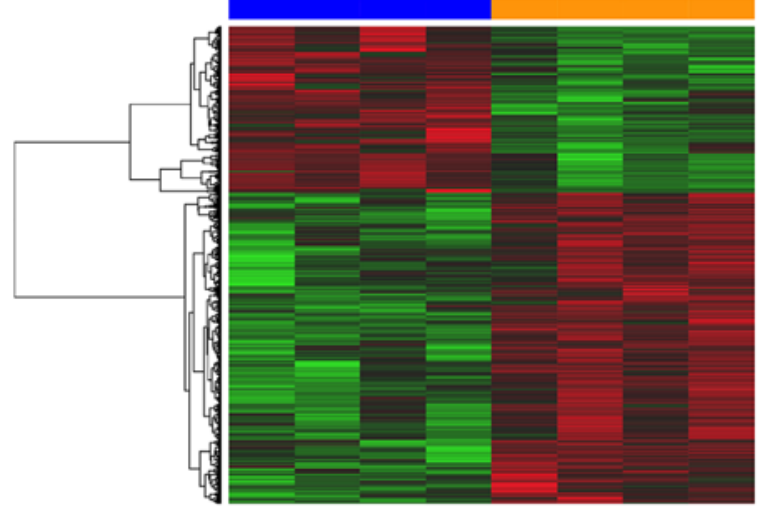

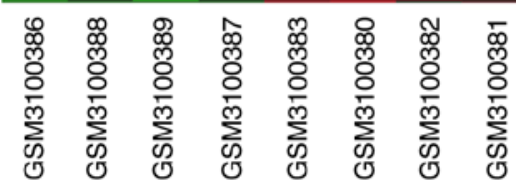

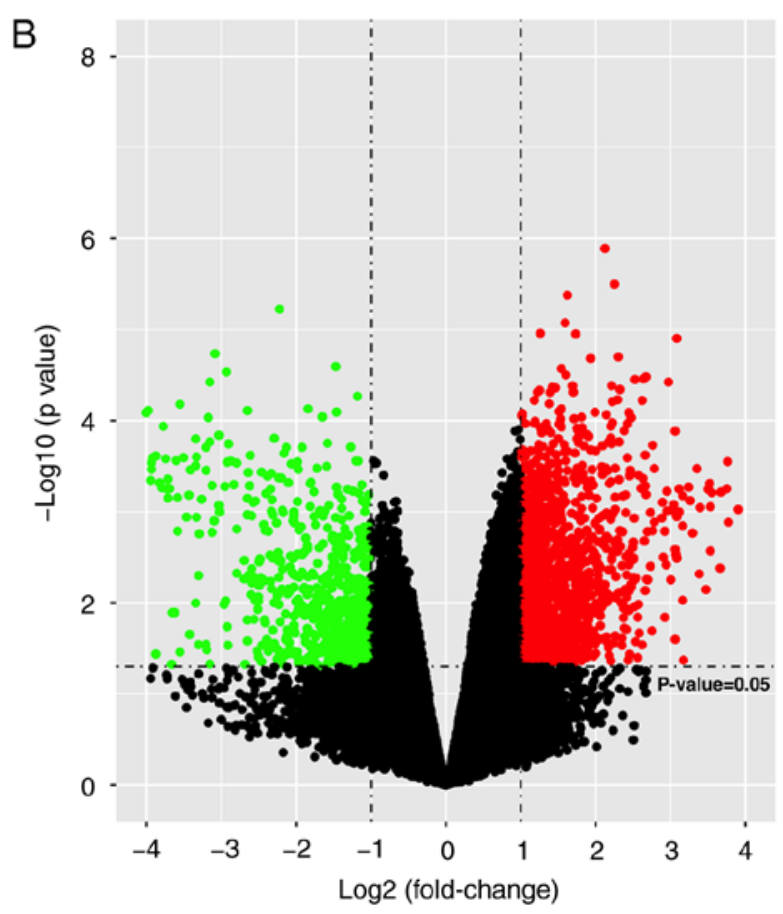

Figure 1. Identification of 2,123 differentially expressed genes. (A) Hierarchical clustering and (B) volcano plot. The black dots in the figure represent genes that were not differentially expressed, the red dots represent upregulated DEGs, and the green points represent downregulated DEGs. DEGs, differentially expressed genes.

\section{Results}

Identification of DEGs. Following data normalization, a differential gene expression analysis was performed using the dataset that included 4 hypertrophic samples and 4 non-hypertrophic control samples. A total of 2,123 DEGs were screened based on values of FDR $<0.05$ and $\log _{2}$ FC $>1$ as the standard. Among these genes, 1,384 genes were upregulated and 739 genes were downregulated. Subsequently, hierarchical clustering was performed based on the DEG expression values from the microarray (Fig. 1A). The differential expression ratios and $\mathrm{P}$-values of the DEGs between each group were also compared using a volcano plot (Fig. 1B).

GO term and KEGG pathway enrichment analysis. To investigate the DEGs on a more functional level, the most recent versions of the GO and KEGG pathway databases were used to analyze the gene symbols and determine the potential functions of the DEGs. GO enrichment analysis revealed that the DEGs were significantly enriched for 742 biological processes (BPs), 277 molecular functions (MFs) and 151 cellular components (CCs). The GO results were mainly associated with collagen in the ECM and included categories, such as ECM organization, collagen-containing ECM, and collagen trimer and integrin binding (Fig. 1A and B). In addition, a total of 311 pathways were potentially involved in the progression of LFH according to the screening results. The KEGG results revealed enrichment mainly in the PI3K/AKT pathway, cytokine-cytokine receptor interactions, protein digestion and absorption, and ECM-receptor interactions (Fig. 2C and D). These pathways have a definite influence on the occurrence and development of LFH, and the PI3K signaling pathway may be an important pathway leading to the development of LFH. Previous studies have reported that PI3K signaling plays an important role in cell proliferation $(23,24)$, which is essential in the development of LFH.

ADAM10 is enriched in the PPI network analysis. According to the PPI pairs in the STRING database, an interaction network of the proteins encoded by the DEGs was constructed. A centrality analysis of the nodes in the PPI network revealed that proenkephalin (PENK), prolyl 4-hydroxylase (P4HB), fibronectin 1 (FN1), fibrinogen alpha chain (FGA), quiescin sulfhydryl oxidase 1 (QSOX1), IL6, serpin family A member 1 (SERPINA1), ADAM10, tissue inhibitor of metalloproteinase 1 (TIMP1) and collagen type I alpha 2 (COL1A2) were crucial genes (Fig. 3). Among these genes, PENK, P4HB, FN1, QSOX1, SERPINA1, ADAM10 and TIMP1 were upregulated in LFH, while FGA, IL6 and COL1A2 were downregulated in LFH. The dysregulation of these genes may be closely related to LFH. Among the genes altered, ADAM10 exhibited significant degrees of correspondence in LFH; this gene has been reported as an important regulator of cell proliferation (25) and plays a regulatory role that is closely related to $\mathrm{PI} 3 \mathrm{~K} / \mathrm{AKT}(26,27)$.

Silencing of ADAM10 inhibits the proliferation of LF cells via the PI3K/AKT pathway. LF cells were cultured and identified. In the present study, LF cells were successfully isolated using an explant method. Within 14 days following explantation, the outgrowth of cells from ligament tissue was observed and became monolayer. Morphologically, the LF cells exhibited features of fibroblast-like cells with a spindle-like shape. As demonstrated by immunofluorescence, the LF cells were positive for vimentin (Fig. 4). To further validate the roles of key 

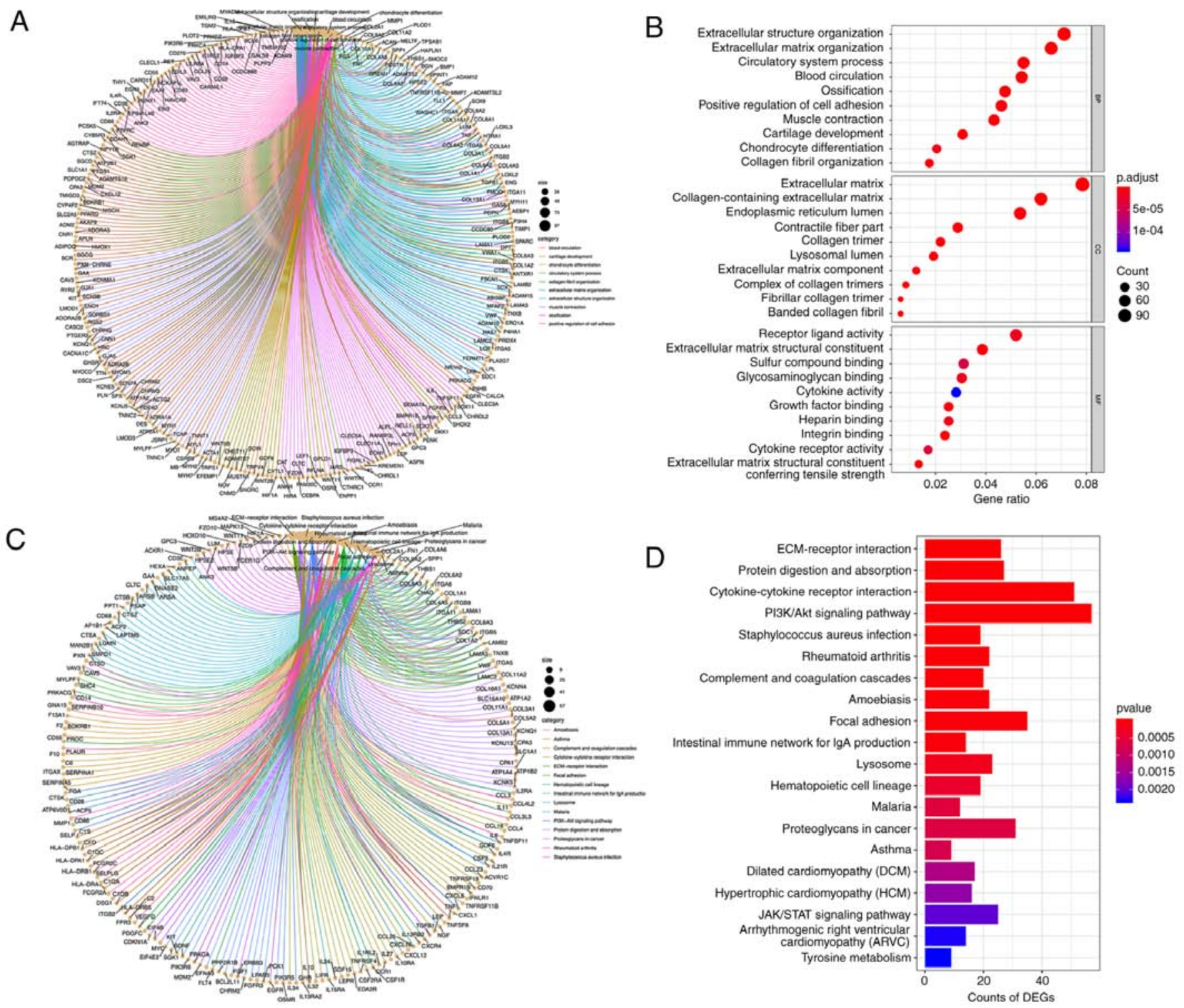

Figure 2. Enrichment analysis of the DEGs. (A and B) GO functional analysis results illustrating the significantly enriched terms for the DEGs. (C and D) Results of the KEGG enrichment analysis of the DEGs. DEGs, differentially expressed genes.

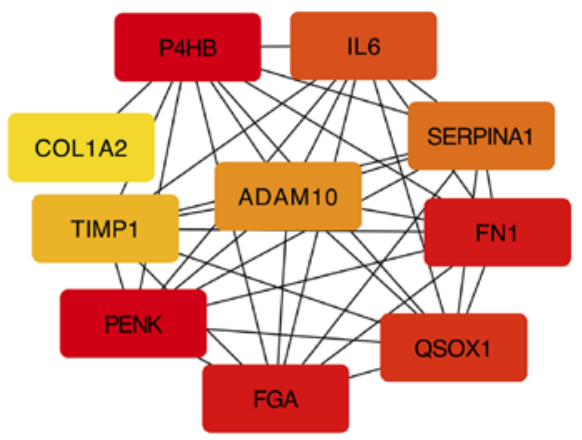

Figure 3. ADAM10 was enriched in the DEGs. The top 10 key genes were screened through the PPI network map. The different colors in the image merely represent different genes and have no other substantial meaning. ADAM10, A disintegrin and metalloproteinase 10; DEGs, differentially expressed genes; PPI, protein-protein interaction.

genes and pathways in the development and progression of $\mathrm{LFH}$, mRNA and protein expression was examined by RT-qPCR and western blot analysis, respectively. The mRNA and protein expression levels of ADAM10 in the ADAM10(-) cells were significantly lower than those in the cells of the controll and sham1 groups $(\mathrm{P}<0.05)$, although significant differences were not observed between the control1 and sham1 group ( $\mathrm{P}>0.05)$. This result indicated that cell transfection-mediated silencing of ADAM10 was successful (Fig. 5A-C). In addition, western blot analysis was used to detect key proteins in the PI3K/AKT signaling pathway. The results revealed that the expression levels of p-AKT and p-PI3K were significantly lower in the ADAM10(-) group compared with the other 2 groups $(\mathrm{P}<0.05$; Fig. 5D-G). Thus, the silencing of ADAM10 downregulated key proteins of the PI3K/AKT pathway.

To further explore the role of the ADAM10 gene in LFH, EdU (Fig. 6A and B) and CCK-8 assays (Fig. 6C) were performed to detect the proliferation of the cells in the 3 groups. In addition, to better verify the role of the PI3K/AKT pathway, the pathway agonist, 740Y-P, was added to the ADAM10(-) cells and the effects on cell proliferation were 
A

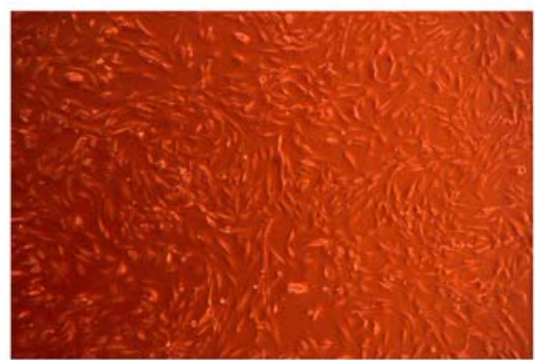

C

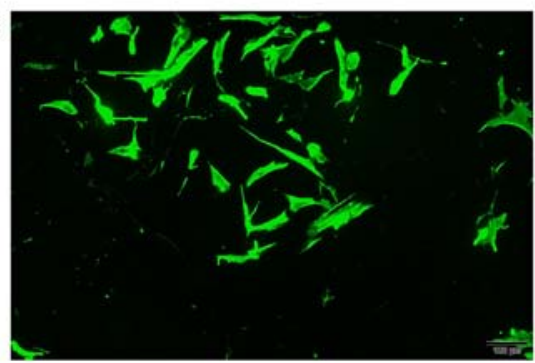

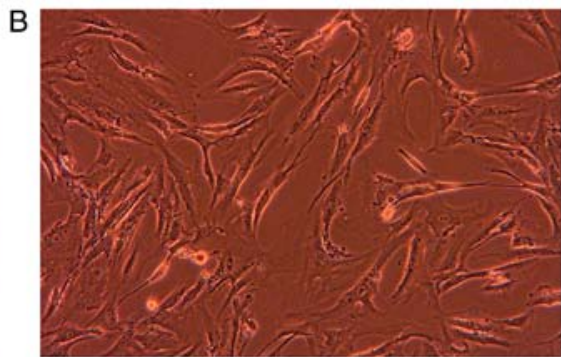

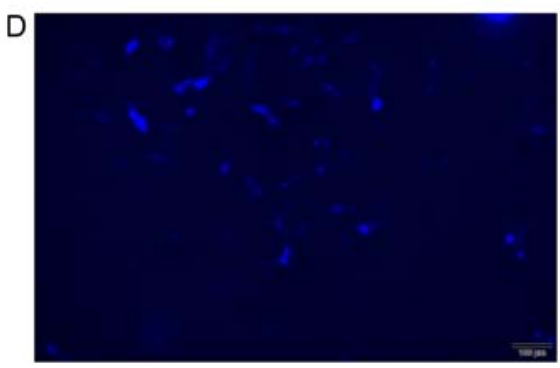

Figure 4. Observation and Immunofluorescence analysis of LF cells. (A) Observation of LF cells under a 40X optical microscope (B) Observation of LF cells under a 100X optical microscope. Immunofluorescence was visualized using (C) FITC (green) and (D) the staining of nuclei with DAPI (blue). LF, ligamentum flavum.

A

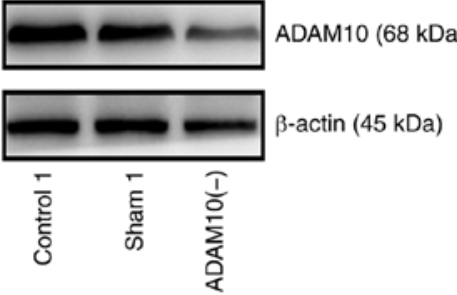

$\mathrm{D}$
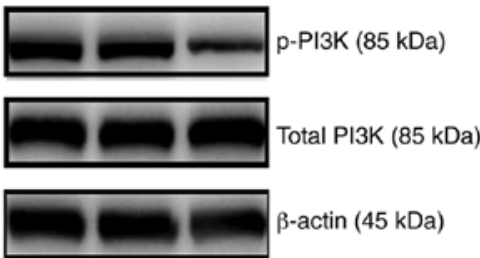

$\mathrm{F}$

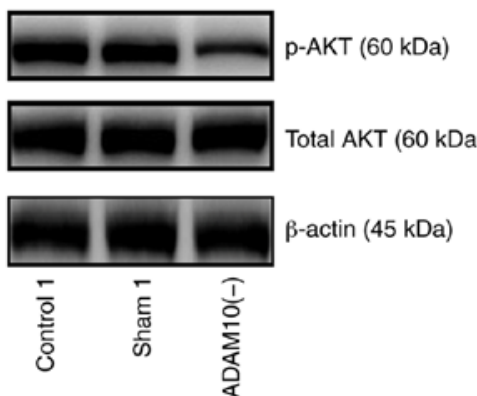

B

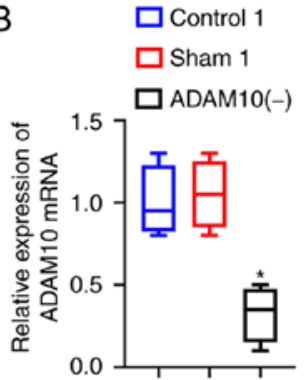

$\mathrm{E}$

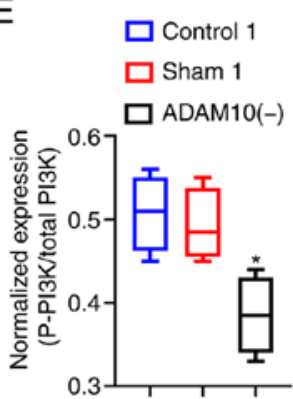

G

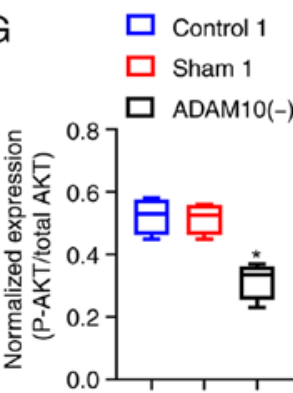

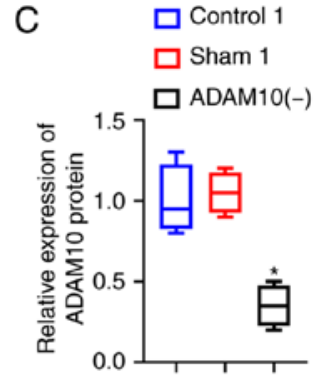

Figure 5. Silencing of ADAM10 inhibits PI3K/AKT pathways (A) Western blot analysis of ADAM10 protein in the 3 groups of cells. (B) RT-qPCR analysis of ADAM10 mRNA in the 3 groups of cells. (C) Western blot analysis of ADAM10 protein in the 3 groups of cells. (D) Western blot analysis of p-PI3K and total PI3K protein in the 3 groups of cells. (E) Western blot analysis of normalized p-PI3K expression (p-PI3K/total PI3K) in the 3 groups of cells. (F) Western blot analysis of p-AKT and total AKT protein in the 3 groups of cells. (G) Western blot analysis of normalized p-AKT expression (p-AKT/total AKT) in the 3 groups of cells. All data are expressed as the means $\pm \mathrm{SD},{ }^{*} \mathrm{P}<0.05$ vs. the control1 and sham 1 groups. ADAM10, A disintegrin and metalloproteinase 10. 
A
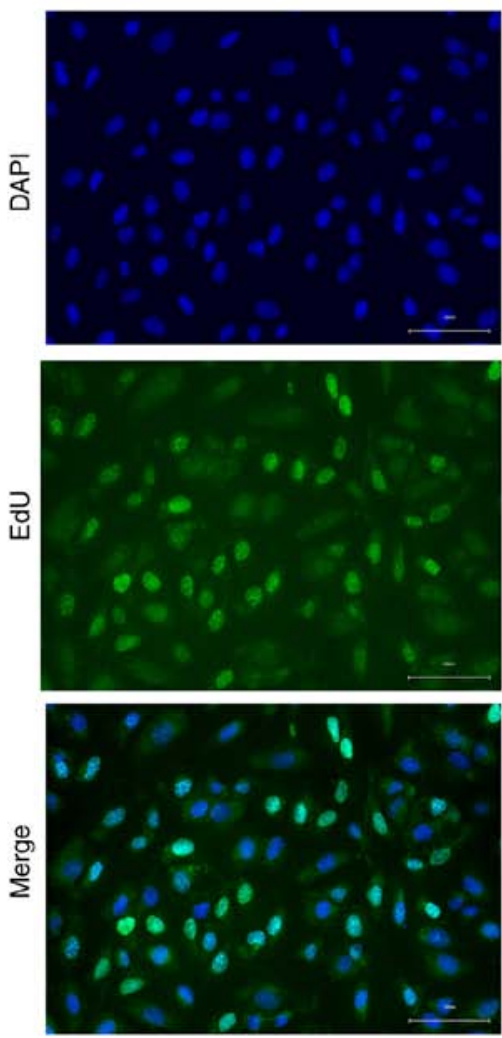

Control
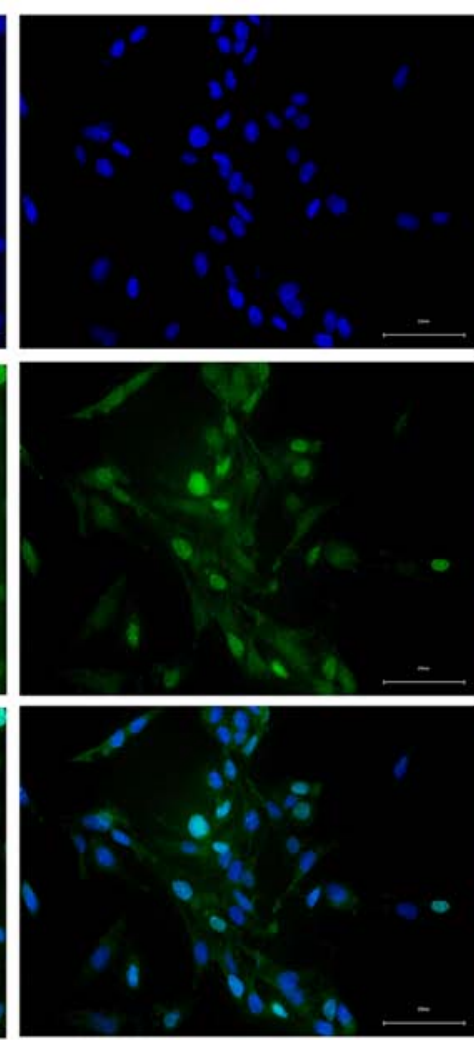

ADAM10(-)
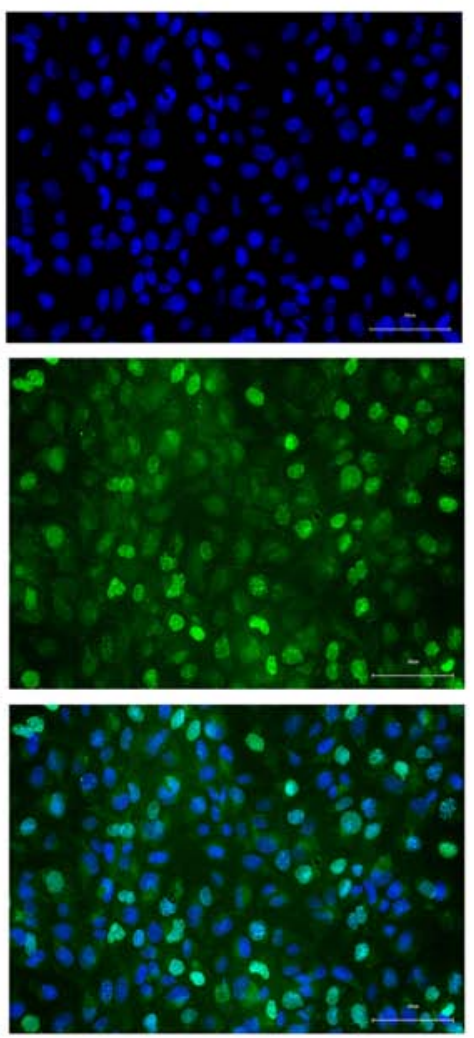

ADAM10(-)+740Y-P
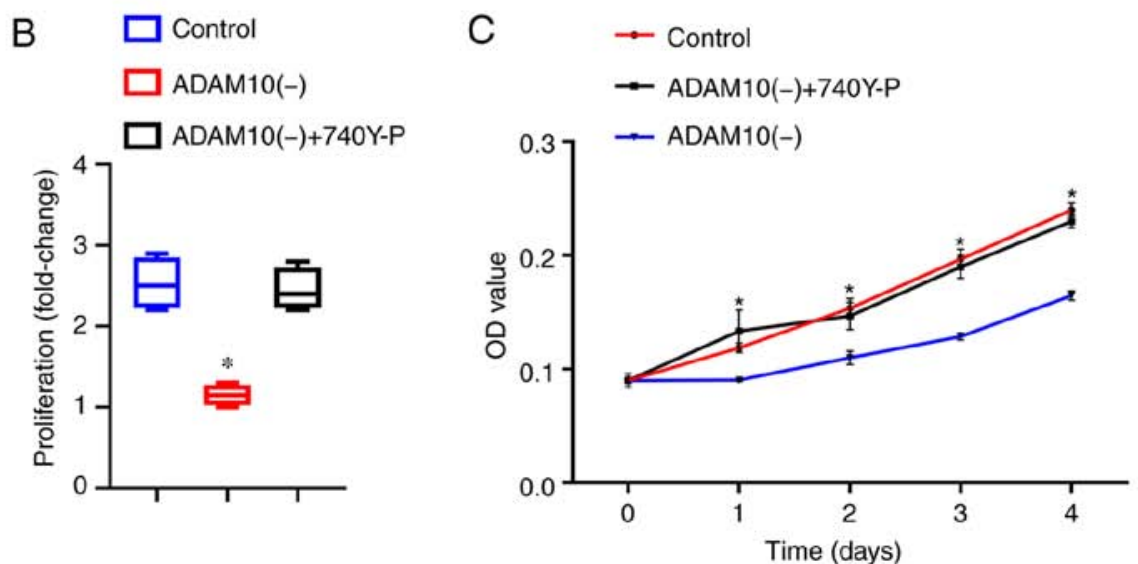

Figure 6. Silencing of ADAM10 inhibit ligamentum cell proliferation via the PI3K/AKT pathway. (A) Results of the EdU incorporation assay illustrating that the proliferative capacity of ligamentum flavum cells was significantly lower in the ADAM10(-) group than the control group and ADAM10(-) + 740Y-P group. However, the proliferative ability did not differ between the control group and the ADAM10(-) + 740Y-P group. (B) Box plot of the EdU results. (C) Results of the CCK-8 assay showing that the OD value was significantly lower in the ADAM10(-) group than the control group and ADAM10(-) + 740Y-P group. However, the OD value did not differ between the control group and ADAM10(-) +740 Y-P group at any time point. All data are expressed as the means \pm SD, ${ }^{*} \mathrm{P}<0.05$ vs. the control and ADAM10(-) + 740Y-P groups. ADAM10, A disintegrin and metalloproteinase 10 .

observed. The results revealed that the proliferation of the ADAM10(-) cells was significantly inhibited compared with the cells in the control group and ADAM10(-) + 740Y-P group $(\mathrm{P}<0.05)$. Moreover, significant differences were not observed between the control group and ADAM10(-) + 740Y-P group. On the whole, the results demonstrated that the silencing of ADAM10 inhibited the proliferation of LF cells via the PI3K/AKT pathway.

Overexpression of ADAM10 increases the proliferation of LF cells via the PI3K/AKT pathway. To further validate the key genes and critical pathways involved in LFH, overexpression experiments were also performed. The mRNA and protein expression levels of ADAM10 in the ADAM10(+) cells were significantly higher than those in the other 2 cell groups $(\mathrm{P}<0.05)$. This result indicated that the overexpression of ADAM10 in LF cells was successful (Fig. 7A-C). Similarly, key proteins in the PI3K/AKT pathway were also detected. The results revealed that the expression levels of $\mathrm{p}-\mathrm{AKT}$ and $\mathrm{p}$-PI3K were significantly higher in the ADAM10(+) cells than the other 2 groups of cells $(\mathrm{P}<0.05$; Fig. 7D-G). 
A

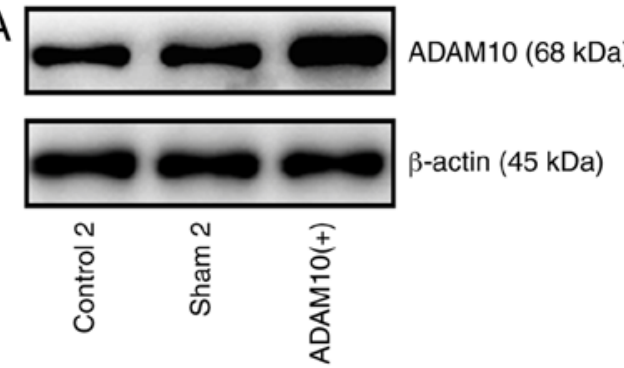

D

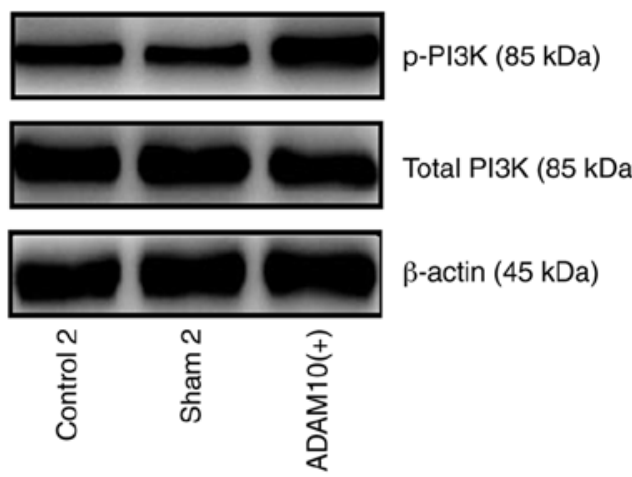

F
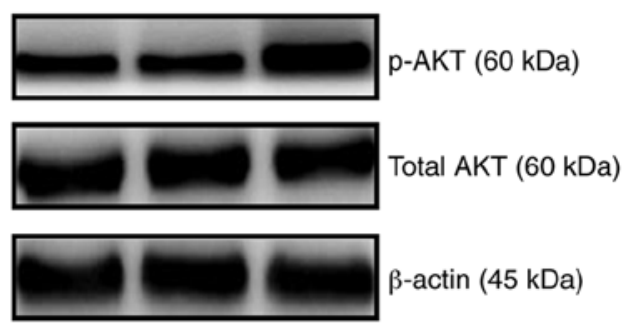

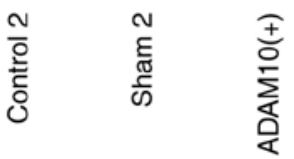

B

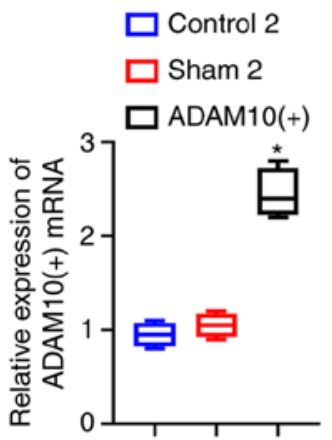

E

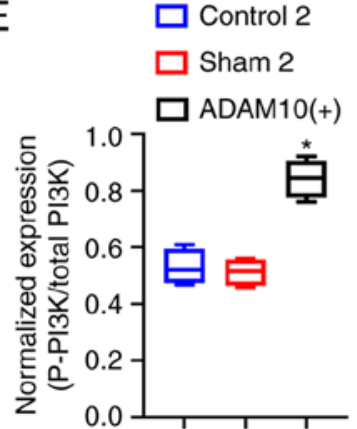

G

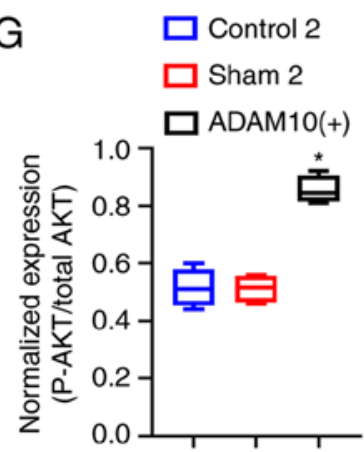

C

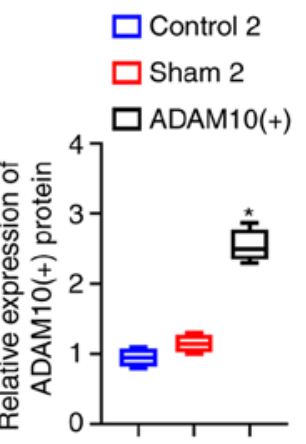

Figure 7. ADAM10 overexpression activates the PI3K/AKT pathway. (A) Western blot band of the ADAM10 protein in the three groups of cells. (B) PCR analysis of the ADAM10 mRNA in the three groups of cells. (C) Western blot analysis of ADAM10 protein in the 3 groups of cells. (D) Western blot analysis of p-PI3K and total PI3K protein in the 3 groups of cells. (E) Western blot analysis of normalized p-PI3K expression (p-PI3K/total PI3K) in the 3 groups of cells. (F) Western blot analysis of the p-AKT and total AKT protein in the 3 groups of cells. (G) Western blot analysis of normalized p-AKT expression (p-AKT/total AKT) in the 3 groups of cells. All data are expressed as the means $\pm \mathrm{SD},{ }^{*} \mathrm{P}<0.05$ vs. the control 2 and sham 2 groups. ADAM10, A disintegrin and metalloproteinase 10 .

To further explore the role of the ADAM10 gene in LFH, EdU (Fig. 8A and B) and CCK-8 assays (Fig. 8C) were performed to detect the proliferation of the cells in the three groups. In addition, to better verify the role of the PI3K/AKT pathway, we added a pathway inhibitor LY294002 to ADAM10(+) cells and observed the effects on cell proliferation. The results revealed that the ADAM10(+) cells exhibited significantly a greater proliferation than the cells in the control and ADAM10(+) + LY294002 group $(\mathrm{P}<0.05)$. Moreover, cell proliferation did not differ between the control and ADAM10(+) + LY294002 groups $(\mathrm{P}>0.05)$. Therefore, it was concluded that ADAM10 promotes the proliferation of LF cells, which leads to hypertrophy of the LF by activating the PI3K/AKT pathway.

\section{Discussion}

Although LF cells are one main factors involved in the pathogenesis of LFH, the cellular mechanisms are not yet completely understood. Therefore, the present study explored the crucial genes and pathways associated with LFH using bioinformatics methods. By comparing the gene expression profiles between $4 \mathrm{LFH}$ tissues and the corresponding non-hypertrophic tissues, 2,123 commonly altered DEGs (1,384 upregulated and 739 downregulated) were identified in LFH tissues.

Furthermore, to better understand the interactions of the identified DEGs, GO functional enrichment and KEGG pathway analyses were performed. The GO analysis revealed that the 2,123 DEGs were mainly associated with the collagen in the ECM, collagen trimer and integrin binding categories. Previous studies have demonstrated that LF fibrosis is characterized by a progressive increase in the presence of collagen in the ECM $(28,29)$. During hypertrophy, the composition of this ECM component is altered and elastic fibers become increasingly fragmented and disordered, and are eventually reduced in number by the overreplacement of collagenous tissue, a process referred to as 'collagen/elastin conversion' $(30,31)$. Fibrosis 
A
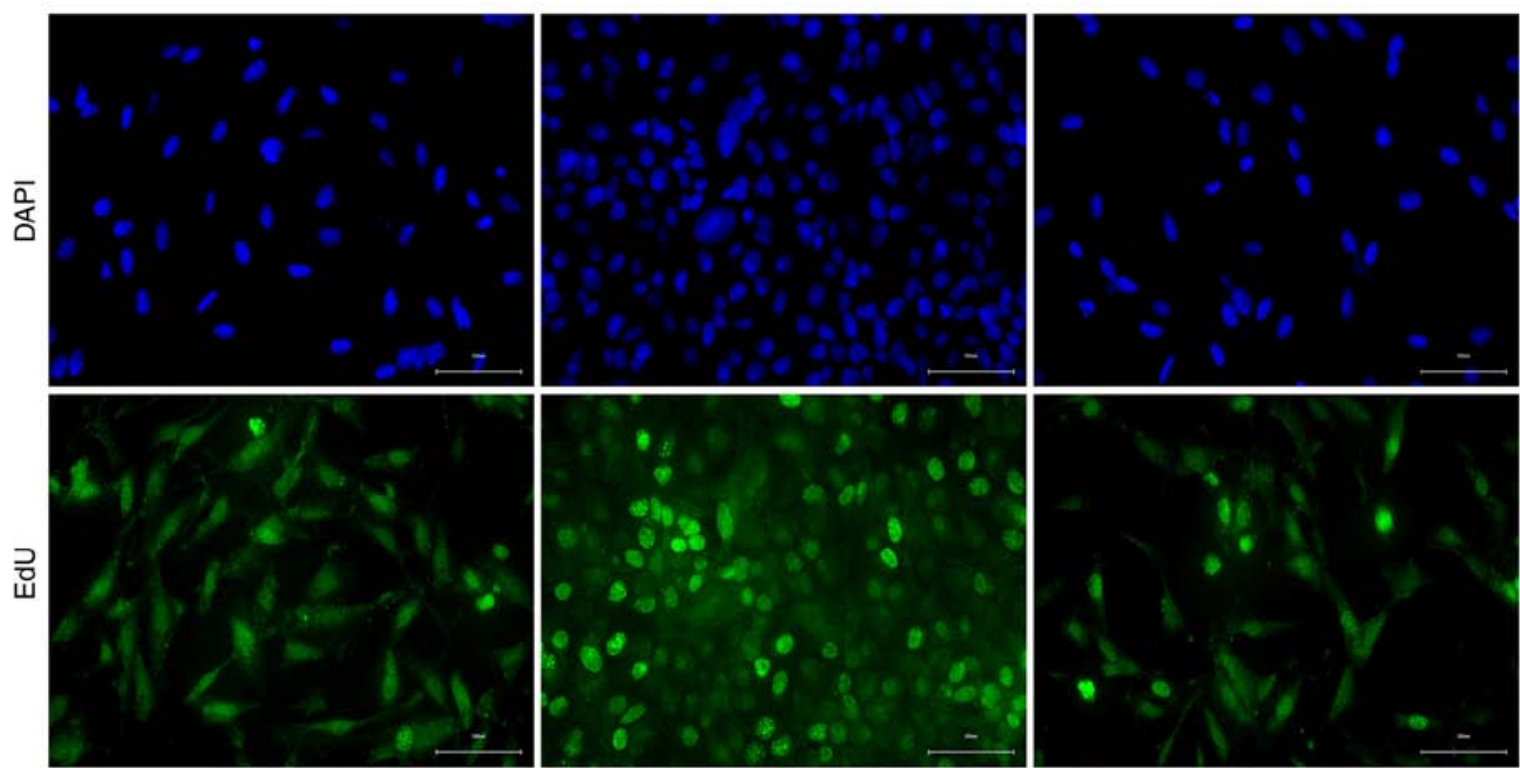

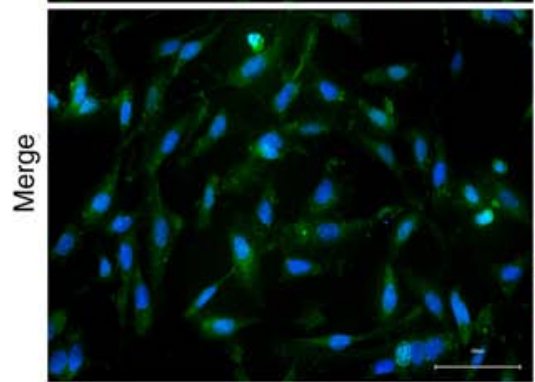

Control

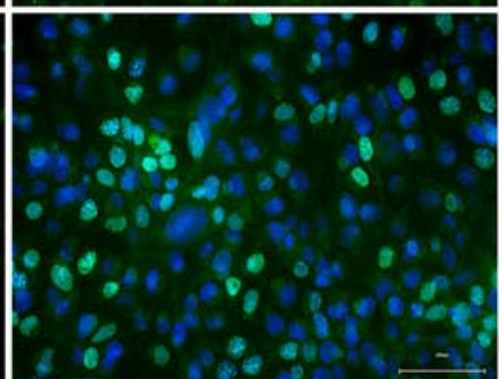

$\operatorname{ADAM} 10(+)$

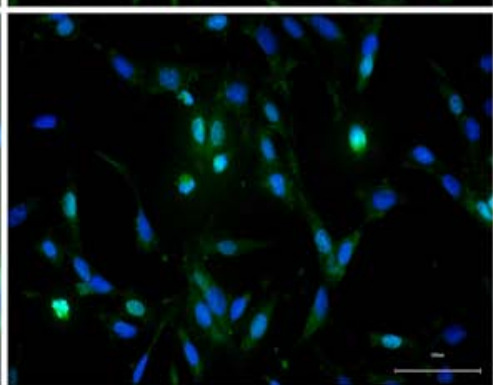

ADAM10(+)+LY294002
B

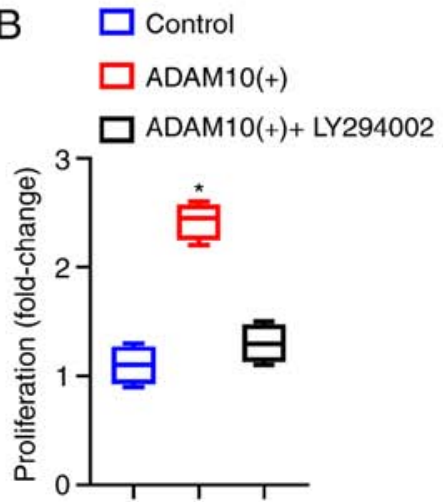

C - Control

$\rightarrow$ ADAM10(+)

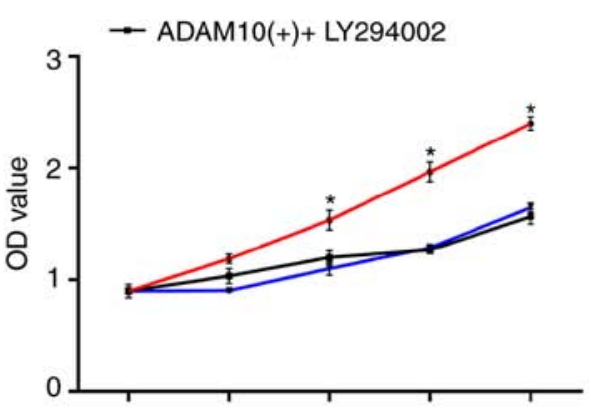

Figure 8. ADAM10 overexpression promotes ligamentum flavum cell proliferation via the PI3K/AKT pathway. (A) Results of the EdU incorporation assay illustrating that the proliferative capacity of ligamentum flavum cells was significantly higher in the ADAM10(+) group than the control group and ADAM10(+) + LY294002 group. However, the proliferative ability did not differ between the control group and ADAM10(+) + LY294002 group. (B) Box plot of the EdU results. (C) Results of the CCK-8 assay showing that the OD value was significantly higher in the ADAM10(+) group than the control group and ADAM10(+) + LY294002 group. However, the OD value did not differ between the control group and ADAM10(+) + LY294002 group at any time. All data are expressed as the means $\pm \mathrm{SD},{ }^{*} \mathrm{P}<0.05$ vs. the control and ADAM10(+) + LY294002 group. ADAM10, A disintegrin and metalloproteinase 10.

leads to excessive fibroblast ECM deposition and tissue remodeling, resulting in a partial loss of tissue structure and organ function. Some studies have suggested that this pathological protein accumulation is due to the excessive recruitment of and increases in the number of fibroblasts (32-34).

KEGG pathway enrichment analysis revealed that the identified DEGs were mainly associated with ECM-receptor interactions, protein digestion and absorption, cytokine-cytokine receptor interactions, the PI3K/AKT signaling pathway and focal adhesion. Among these pathways, the PI3K/AKT pathway is of particular importance for cell membrane receptor signaling. After specific ligands are bound to the cell membrane, signals are transmitted through a cascade of AKT, JNK and Ras, which affects cell proliferation, migration and apoptosis (35). Following PI3K activation, AKT can activate certain cyclins and quiescent cells to accelerate cell cycle progression, thus promoting cell proliferation $(36,37)$. In the present study, the PI3K/AKT pathway was highly associated with LFH tissue compared with normal LF tissue. This result indicates that the PI3K/AKT pathway plays an important role in the pathological development of LFH. 
According to the centrality of the nodes in the PPI network, crucial DEGs, including PENK, P4HB, FN1, FGA, QSOX1, IL6, SERPINA1, ADAM10, TIMP1 and COL1A2 were identified. Among these genes, PENK, P4HB, FN1, QSOX1, SERPINA1, ADAM10 and TIMP1 were upregulated in LFH, while FGA, IL6 and COL1A2 were downregulated. Inhibiting or promoting these genes may be a therapeutic strategy for the treatment of LH. Among these genes, ADAM10 was upregulated; the ADAM family is one of the major families of proteases that act as sheddases, which play crucial roles in activating or inhibiting some biomolecular processes $(38,39)$. These proteases are characterized by a modular domain structure consisting of an N-terminal signal sequence followed by a pro-domain, a metalloprotease (catalytic) domain, a dissociation domain, an epidermal growth factor-like (cysteine-rich) domain, a single transmembrane domain and a cytoplasmic fraction $(40,41)$. ADAM10 is involved in the shedding of a number of substrates that are critical for cancer progression, neurological and vascular diseases, degenerative diseases, and the morphogenesis and the remodeling of some tissues (42-46). A recent study on pulmonary fibrosis revealed that ADAM10 is the major metalloproteinase in human lung fibroblasts and increases in its expression can continue to activate fibroblast proliferation and muscle formation to promote organ fibrosis (47). Research into epithelial tissues has found that ADAM10 plays important roles in cell development, cell detachment, cell proliferation and cell survival during development and under pathological conditions (25). In addition, ADAM10 is closely related to the PI3K/AKT pathway. Moreover, studies have demonstrated that ADAM10 can promote cell proliferation by activating this pathway $(26,27,48)$.

The present study puts forth a number of important insights. First, microarray and bioinformatics analyses were applied to analyze whole transcriptome changes between normal LF and LFH tissue, and the genetic mechanisms underlying LFH was thoroughly and accurately analyzed. Second, the present study combined bioinformatics methods and molecular biology experiments to examine the molecular mechanisms of LFH, which not only reduced the false-positive rate of bioinformatics methods, but also compensated for the limitations of molecular biology experiments. Third, the present study provides a new direction for future research on the mechanisms of LFH and provides a novel approach for the clinical treatment and prevention of diseases related to LFH.

However, there is also a limitation tp the present study. Datasets, such as the GEO database, The Cancer Genome Atlas (TCGA) database, the ArrayExpress database and InSilicoDB were searched. However, there are no data related to LFH apart from the GEO database used in the current study. As a result, only 4 LFH samples and 4 normal samples were included in the present cohort. The authors aim to use more samples and perform further experiments to confirm the results and conclusions obtained herein in future studies.

In conclusion, the present study suggests that ADAM10 promotes the proliferation of LF cells by activating the PI3K/AKT pathway, which may be a pathogenic mechanism of LFH.

\section{Acknowledgements}

Not applicable.

\section{Funding}

The present study was supported by grants from the Youth Program of National Natural Science Foundation of China (no. 81801213), China Postdoctoral Science Foundation, No. 65 General Fund (no. 2019M651967), Jiangsu Planned Projects for Postdoctoral Research Funds (no. 2018K176C) and the China Postdoctoral start-up fund (no. 2018107007).

\section{Availability of data and materials}

All data generated or analyzed during this study are included in this published article.

\section{Authors' contributions}

FY and KG designed the present study. BP, TH and MC performed the experiments. LJ, HF, ZQ and XL analyzed the data and prepared the figures. BP and TH drafted the initial manuscript. BP and TH reviewed and revised the manuscript. All authors read and approved the manuscript and agree to be accountable for all aspects of the research in ensuring that the accuracy or integrity of any part of the work are appropriately investigated and resolved.

\section{Ethics approval and consent to participate}

The study was approved by the Ethics Committee of Xuzhou Medical University Affiliated Hospital and written consent was obtained from all patients.

\section{Patient consent for publication}

Not applicable.

\section{Competing interests}

The authors declare that they have no competing interests.

\section{References}

1. Sakai Y, Ito S, Hida T, Ito K, Harada A and Watanabe K: Clinical outcome of lumbar spinal stenosis based on new classification according to hypertrophied ligamentum flavum. J Orthop Sci 22: 27-33, 2017.

2. Delen E, Doğanlar O, Delen Ö, Doğanlar ZB and Kılınçer C: The role of JAK-STAT signaling activation in hypertrophied ligamentum flavum. World Neurosurg 137: e506-e516, 2020.

3. Ciol MA, Deyo RA, Howell E and Kreif S: An assessment of surgery for spinal stenosis: Time trends, geographic variations, complications, and reoperations. J Am Geriatr Soc 44: 285-290, 1996.

4. Sun C, Liu X, Guan G and Zhang H: Increased expression of WISP-1 (CCN4) contributes to fibrosis in the hypertrophied lumber ligamentum flavum. Int J Clin Exp Pathol 10: 1356-1363, 2017.

5. Park JO, Lee BH, Kang YM, Kim TH, Yoon JY, Kim H, Kwon UH, Lee KI, Lee HM and Moon SH: Inflammatory cytokines induce fibrosis and ossification of human ligamentum flavum cells. J Spinal Disord Tech 26: E6-E12, 2013.

6. Hur JW, Bum-Joon K, Jin-Hyun P, Kim JH, Park YK, Kwon TH and Moon HJ: The mechanism of ligamentum flavum hypertrophy: Introducing angiogenesis as a critical link that couples mechanical stress and hypertrophy. Neurosurgery 77: 281-282, 2015.

7. Kang YM, Suk KS, Lee BH, Kim HS, Lee KI, Park SY, Lee HM and Moon SH: Herniated intervertebral disk induces hypertrophy and ossification of ligamentum flavum. J Spinal Disord Tech 27: 382-389, 2014. 
8. Nakatani T, Marui T,Hitora T,Doita M, Nishida K and Kurosaka M: Mechanical stretching force promotes collagen synthesis by cultured cells from human ligamentum flavum via transforming growth factor-betal. J Orthop Res 20: 1380-1386, 2002.

9. Amudong A, Muheremu A and Abudourexiti T: Hypertrophy of the ligamentum flavum and expression of transforming growth factor beta. J Int Med Res 45: 2036-2041, 2017.

10. Sun C, Wang Z, Tian JW and Wang YH: Leptin-Induced inflammation by activating IL-6 expression contributes to the fibrosis and hypertrophy of ligamentum flavum in lumbar spinal canal stenosis. Biosci Rep 38: BSR20171214, 2018.

11. Sairyo K, Biyani A, Goel V, Leaman D, Booth R Jr, Thomas J, Gehling D, Vishnubhotla L, Long $\mathrm{R}$ and Ebraheim $\mathrm{N}$ : Pathomechanism of ligamentum flavum hypertrophy: A multidisciplinary investigation based on clinical, biomechanical, histologic, and biologic assessments. Spine (Phila Pa 1976) 30 2649-2656, 2005.

12. Sairyo K, Biyani A, Goel VK, Leaman DW, Booth R Jr, Thomas J, Ebraheim NA, Cowgill IA and Mohan SE: Lumbar ligamentum flavum hypertrophy is due to accumulation of inflammation-related scar tissue. Spine (Phila Pa 1976) 32 E340-E347, 2007.

13. Yücetaş SC and Çakir T: Decreased catalase expression is associated with ligamentum flavum hypertrophy due to lumbar spinal canal stenosis. Medicine (Baltimore) 98: e15192, 2019.

14. Chen J, Liu Z, Zhong G, Qian L, LiZ, Qiao Z, Chen B and Wang H: Hypertrophy of ligamentum flavum in lumbar spine stenosis is associated with increased miR-155 level. Dis Markers 2014 $786543,2014$.

15. Diboun I, Wernisch L, Orengo CA and Koltzenburg M: Microarray analysis after RNA amplification can detect pronounced differences in gene expression using limma. BMC Genomics 7: 252, 2006

16. Irizarry RA, Hobbs B, Collin F, Beazer-Barclay YD, Antonellis KJ, Scherf U and Speed TP: Exploration, normalization, and summaries of high density oligonucleotide array probe level data. Biostatistics 4: 249-264, 2003.

17. Yu G, Wang LG, Han Y and He QY: ClusterProfiler: An R package for comparing biological themes among gene clusters. Omics 16: 284-287, 2012

18. Shannon P, Markiel A, Ozier O, Baliga NS, Wang JT, Ramage D, Amin N, Schwikowski B and Ideker T: Cytoscape: A software environment for integrated models of biomolecular interaction networks. Genome Res 13: 2498-2504, 2003.

19. Tang Y, Li M, Wang J, Pan Y and Wu FX: CytoNCA: A cytoscape plugin for centrality analysis and evaluation of protein interaction networks. Biosystems 127: 67-72, 2015.

20. Specchia N, Pagnotta A, GiganteA, Logroscino G and Toesca A: Characterization of cultured human ligamentum flavum cells in lumbar spine stenosis. J Orthop Res 19: 294-300, 2001.

21. Yan B, Huang M, Zeng C, Yao N, Zhang J, Yan B, Jiang H, Tian X, Ao X, Zhao H, et al: Locally produced IGF-1 Promotes hypertrophy of the ligamentum flavum via the mTORC1 signaling pathway. Cell Physiol Biochem 48: 293-303, 2018.

22. Livak KJ and Schmittgen TD: Analysis of relative gene expression data using real-time quantitative PCR and the 2(-Delta Delta C(T)) method. Methods 25: 402-408, 2001

23. Okada M, Oba Y and Yamawa H: Endostatin stimulates proliferation and migration of adult rat cardiac fibroblasts through PI3K/Akt pathway. Eur J Pharmacol 750: 20-26, 2015.

24. Feng X, Wu C, Yang M, Liu Q, Li H, Liu J, Zhang Y, Hao Y, Kang L, Zhang Y and Liu S: Role of PI3K/Akt signal pathway on proliferation of mesangial cell induced by HMGB1. Tissue Cell 48: 121-125, 2016.

25. Maretzky T, Reiss K, Ludwig A, Buchholz J, Scholz F, Proksch E, de Strooper B, Hartmann D and Saftig P: ADAM10 mediates E-cadherin shedding and regulates epithelial cell-cell adhesion, migration, and beta-catenin translocation. Proc Natl Acad Sci USA 102: 9182-9187, 2005.

26. Boccalini G, Sassoli C, Bani D and Nistri S: Relaxin induces up-regulation of ADAM10 metalloprotease in RXFP1-expressing cells by PI3K/AKT signaling. Mol Cell Endocrinol 472: 80-86, 2018.

27. Li D, Xiao Z, Wang G and Song X: Knockdown of ADAM10 inhibits migration and invasion of fibroblast-like synoviocytes in rheumatoid arthritis. Mol Med Rep 12: 5517-5523, 2015.

28. Zhang K, Sun W, Liu XY, Zhao CQ, Li H, Sun XJ, You-Zhuan X, Ding W and Zhao J: Hypertrophy and fibrosis of the ligamentum flavum in lumbar spinal stenosis is associated with increased expression of LPA and LPAR1. Clin Spine Surg 30: E189-E191, 2017.
29. Adair-Kirk TL and Senior RM: Fragments of extracellular matrix as mediators of inflammation. Int $\mathrm{J}$ Biochem Cell Biol 40: 1101-1110, 2008.

30. Yoshida M, Shima K, Taniguchi Y, Tamaki T and Tanaka T: Hypertrophied ligamentum flavum in lumbar spinal canal stenosis. Pathogenesis and morphologic and immunohistochemical observation. Spine 17: 1353-1360, 1992.

31. Kosaka H, Sairyo K, Biyani A, Leaman D, Yeasting R, Higashino K, Sakai T, Katoh S, Sano T, Goel VK and Yasui N: Pathomechanism of loss of elasticity and hypertrophy of lumbar ligamentum flavum in elderly patients with lumbar spinal canal stenosis. Spine (Phila Pa 1976) 32: 2805-2811, 2007.

32. Chambers RC and Mercer PF: Mechanisms of alveolar epithelial injury, repair, and fibrosis. Ann Am Thorac Soc 12 (Suppl 1): S16-S20, 2015

33. Duffield JS: Cellular and molecular mechanisms in kidney fibrosis. J Clin Invest 124: 2299-2306, 2014.

34. Park JB, Lee JK, Park SJ and Riew KD: Hypertrophy of ligamentum flavum in lumbar spinal stenosis associated with increased proteinase inhibitor concentration. J Bone Joint Surg Am 87: 2750-2757, 2005

35. Matsuoka $\mathrm{T}$ and Yashiro M: The role of PI3K/Akt/mTOR signaling in gastric carcinoma. Cancers (Basel) 6: 1441-1463, 2014.

36. Lin Z, Zhou P, von Gise A, Gu F, Ma Q, Chen J, Guo H, van Gorp PR, Wang DZ and Pu WT: Pi3kcb links hippo-YAP and PI3K-AKT signaling pathways to promote cardiomyocyte proliferation and survival. Circ Res 116: 35-45, 2015.

37. Meng F, Wang F, Wang L, Wong S, Cho WC and Chan LW: MiR-30a-5p overexpression may overcome EGFR-inhibitor resistance through regulating PI3K/AKT signaling pathway in non-small cell lung cancer cell lines. Front Genet 7: 197, 2016.

38. Edwards DR, Handsley MM and Pennington CJ: The ADAM metalloproteinases. Mol Aspects Med 29: 258-289, 2008.

39. Pham DH, Kim JS, Kim SK, Shin DJ, Uong NT, Hyun H, Yoon MS, Kang SJ, Ryu YJ, Cho JS, et al: Effects of ADAM10 and ADAM17 inhibitors on natural killer cell expansion and antibody-dependent cellular cytotoxicity against breast cancer cells in vitro. Anticancer Res 37: 5507-5513, 2017.

40. Klein T and Bischoff R: Active metalloproteases of the A disintegrin and metalloprotease (ADAM) family: Biological function and structure. J Proteome Res 10: 17-33, 2011.

41. Scheller J, Chalaris A, Garbers C and Rose-John S: ADAM17: A molecular switch to control inflammation and tissue regeneration. Trends Immunol 32: 380-387, 2011.

42. Bae WY, Park SK, Kim DH, Koh TK, Hur DY and Chueh HW: Expression of ADAM17 and ADAM10 in nasal polyps. Int Forum Allergy Rhinol 6: 731-736, 2016.

43. Matthews AL, Noy PJ, Reyat JS and Tomlinson MG: Regulation of A disintegrin and metalloproteinase (ADAM) family sheddases ADAM10 and ADAM17: The emerging role of tetraspanins and rhomboids. Platelets 28: 333-341, 2017.

44. Pruessmeyer J and Ludwig A: The good, the bad and the ugly substrates for ADAM10 and ADAM17 in brain pathology, inflammation and cancer. Semin Cell Dev Biol 20: 164-174, 2009.

45. Chueh HW, Park SK, Hur DY and Bae WY: Expression profile of ADAM10 and ADAM17 in allergic rhinitis. Int Forum Allergy Rhinol 5: 1036-1041, 2015.

46. Huang WH, Chen W, Jiang LY, Yang YX, Yao LF and Li KS: Influence of ADAM10 polymorphisms on plasma level of soluble receptor for advanced glycation end products and the association with alzheimer's disease risk. Front Genet 9: 540, 2018.

47. Lagares D, Ghassemi-Kakroodi P, Tremblay C, Santos A, Probst CK, Franklin A, Santos DM, Grasberger P, Ahluwalia N, Montesi SB, et al: ADAM10-Mediated ephrin-B2 shedding promotes myofibroblast activation and organ fibrosis. Nat Med 23: 1405-1415, 2017.

48. Liu S, Zhang W, Liu K, Ji B and Wang G: Silencing ADAM10 inhibits the in vitro and in vivo growth of hepatocellular carcinoma cancer cells. Mol Med Rep 11: 597-602, 2015.

This work is licensed under a Creative Commons Attribution-NonCommercial-NoDerivatives 4.0 International (CC BY-NC-ND 4.0) License. 